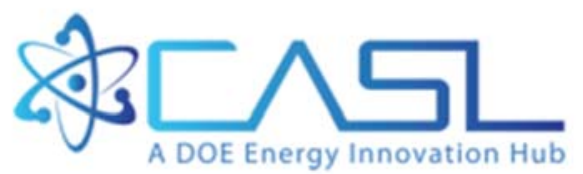

CASL-U-2016-1170-000

\title{
VERA 3.5 Installation Guide
}

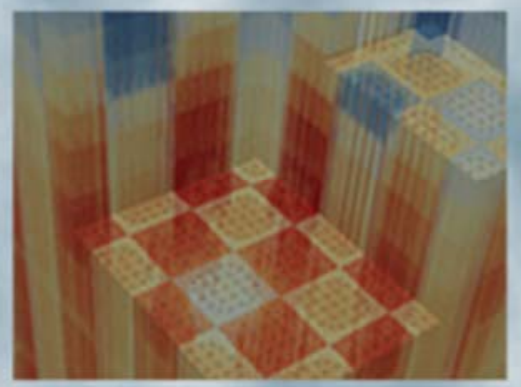

August 16, 2016
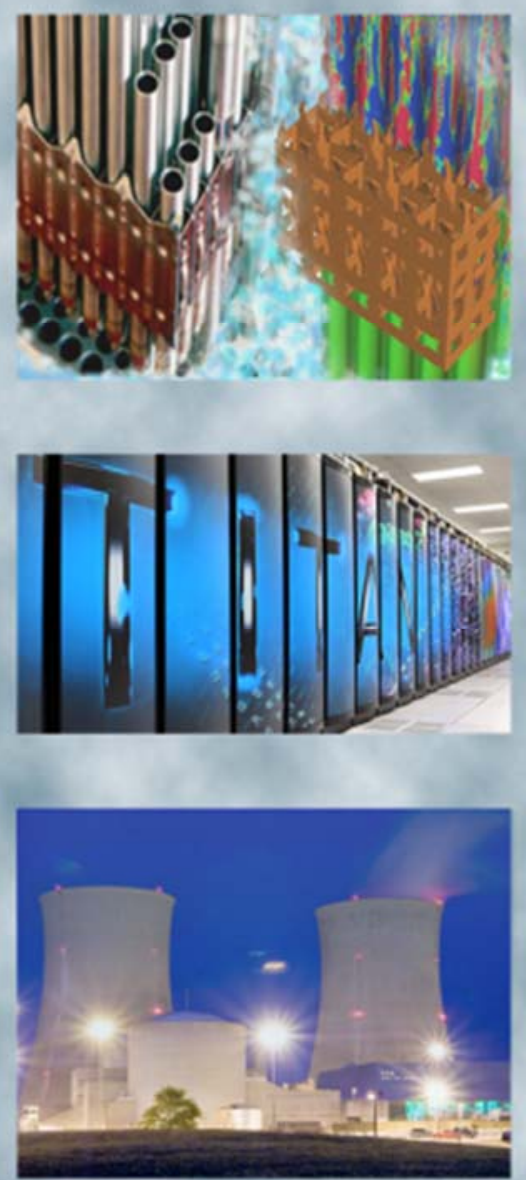


\section{REVISION LOG}

\begin{tabular}{|c|c|c|l|}
\hline Revision & Date & Affected Pages & \multicolumn{1}{c|}{ Revision Description } \\
\hline 0 & & All & Initial Version \\
\hline & & & \\
\hline & & & \\
\hline & & & \\
\hline
\end{tabular}

Document pages that are:

Export Controlled ____ None

IP/Proprietary/NDA Controlled None

Sensitive Controlled___ None

\section{Requested Distribution:}

To: Unlimited distribution

Copy: 


\title{
DOCUMENT AVAILABILITY
}

Reports produced after January 1, 1996, are generally available free via US Department of Energy (DOE) SciTech Connect.

Website http://www.osti.gov/scitech/

Reports produced before January 1, 1996, may be purchased by members of the public from the following source:

\author{
National Technical Information Service \\ 5285 Port Royal Road \\ Springfield, VA 22161 \\ Telephone 703-605-6000 (1-800-553-6847) \\ TDD 703-487-4639 \\ Fax 703-605-6900 \\ E-mail info@ntis.gov \\ Website http://www.ntis.gov/help/ordermethods.aspx
}

Reports are available to DOE employees, DOE contractors, Energy Technology Data Exchange representatives, and International Nuclear Information System representatives from the following source:

Office of Scientific and Technical Information

PO Box 62

Oak Ridge, TN 37831

Telephone 865-576-8401

Fax 865-576-5728

E-mail reports@osti.gov

Website http://www.osti.gov/contact.html

This report was prepared as an account of work sponsored by an agency of the United States Government. Neither the United States Government nor any agency thereof, nor any of their employees, makes any warranty, express or implied, or assumes any legal liability or responsibility for the accuracy, completeness, or usefulness of any information, apparatus, product, or process disclosed, or represents that its use would not infringe privately owned rights. Reference herein to any specific commercial product, process, or service by trade name, trademark, manufacturer, or otherwise, does not necessarily constitute or imply its endorsement, recommendation, or favoring by the United States Government or any agency thereof. The views and opinions of authors expressed herein do not necessarily state or reflect those of the United States Government or any agency thereof. 


\section{VERA Installation Guide}

Author: Roscoe A. Bartlett (bartlettra@ornl.gov)

Author: Mark Baird (bairdml@ornl.gov)

Author: Mark Berrill (berrillma@ornl.gov)

Author: Joel A. Kulesza (jkulesza@umich.edu)

Author: Brenden T. Mervin (bmervin@epri.com)

Contact: support@casl.gov

Date: $\quad 2016-08-29$

Version: vera-3.5.0-dev-103-gf7c9388

\section{Contents}

1 Introduction 1

2 Standard VERA Dev Env Directory Structure 1

3 Installation Process 3

3.1 Make sure the basic prerequisites are satisfied $\ldots \ldots \ldots \ldots$

3.2 Determine source, scratch and install directories . . . . . . . . . . . . . . . . . . . 4

3.3 Get the base VERA and TriBITS source directories . . . . . . . . . . . . . . . . . . . . 4

3.4 Install the base development environment . . . . . . . . . . . . . . . . . . . . . . 4

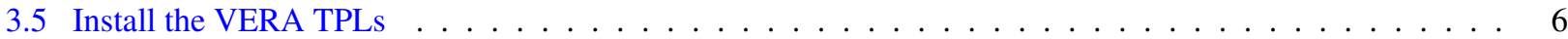

3.6 Build and build/install VERA Components . . . . . . . . . . . . . . . . . . . . . . . 6

3.7 Final setup of installed VERA dev env and final cleanup . . . . . . . . . . . . . . . . . . . . 7

4 Details on Initial Setup $\quad 8$

4.1 Requesting Access to VERA Repositories . . . . . . . . . . . . . . . . . . . 8

4.2 System Configuration Considerations . . . . . . . . . . . . . . . . . . . . . . . 8

4.3 Minimal System Package Setup . . . . . . . . . . . . . . . . . . . . . . . 8

4.4 SSH Setup For Accessing casl-dev _ . . . . . . . . . . . . . . . . . . . . . . . . . 9

4.5 Create Unix User and Group . . . . . . . . . . . . . . . . . . . . . . . . . 10

4.6 Setup Base Directories for VERA . . . . . . . . . . . . . . . . . . . . . . . . 10

5 Details on TPL Installation $\quad 11$

6 Details VERA Component Build, Test, and Installation 12

6.1 Load VERA Dev Env . . . . . . . . . . . . . . . . . . . . . . . . . . . 12

6.2 Clone Remaining VERA Components . . . . . . . . . . . . . . . . . . . 12

6.3 Checking Out a Specific Version of VERA . . . . . . . . . . . . . . . . . . . . 13

7 Details on Finalizing VERA Dev Env Installation 15 
8.1 Get Source For VERA Components To Install _ . . . . . . . . . . . . . . . . . . . . . . . . 16

8.2 Configure, Build, And Test VERA Components To Install . . . . . . . . . . . . . . . . . . . . 16

8.3 Install Built VERA Components . . . . . . . . . . . . . . . . . . . . 17

8.4 Documentation For Installed VERA Components ． . . . . . . . . . . . . . . . . . . 17

9 Appendix 17

9.1 Set Up Remote SSH Tunnel . . . . . . . . . . . . . . . . . . . . . . . . . . . . 17

9.2 Minimal System Package Setup on Various Systems ． . . . . . . . . . . . . . . . . . . . . 18

9.3 Official VERA TPL Versions . . . . . . . . . . . . . . . . . . . . . . . 19

9.4 Shared versus Static Libraries . . . . . . . . . . . . . . . . . . . . . . . . . . 20

\section{Introduction}

This guide describes the structure and setup of the standard VERA development environment (VERA Dev Env) and standard VERA Third Party Libraries (TPLs) that need to be in place before installing many of the VERA simulation components. It describes everything from the initial setup on a new machine to the final build, testing, and installation of VERA components. The goal of this document is to describe how to create the directories and contents outlined in Standard VERA Dev Env Directory Structure and then obtain the remaining VERA source and build, test, and install any of the necessary VERA components on a given system. This document describes the process both for a development version of VERA and for a released tarball of the VERA sources.

One should start by getting acquainted with Standard VERA Dev Env Directory Structure. Then one should work through the Installation Process to see the major steps needed. If everything goes well, the Installation Process contains all of the information needed to perform the full install of the VERA Dev Env as well as the install of VERA components themselves. The remaining sections contain more information and details for variations and tips for how to solve problems when things go wrong.

WARNING: This guide only describes the installation of the VERA Dev Env and TPLs and does not contain specific information about specific VERA simulation components. That information is found in other sources. Please consult with a CASL representative about what VERA components are available to install from source and what capabilities they provide. Mention of other VERA repositories in only used as examples and may not even be up to date.

\section{Standard VERA Dev Env Directory Structure}

The standard directory structure for the installation of the VERA Development Environment (VERA Dev Env) is given below:

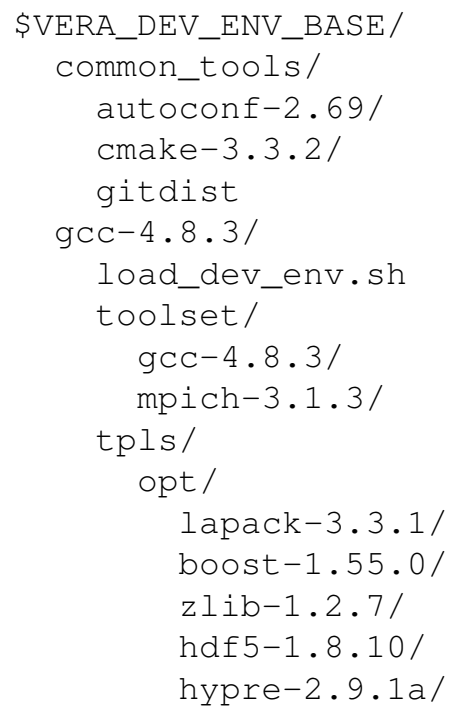




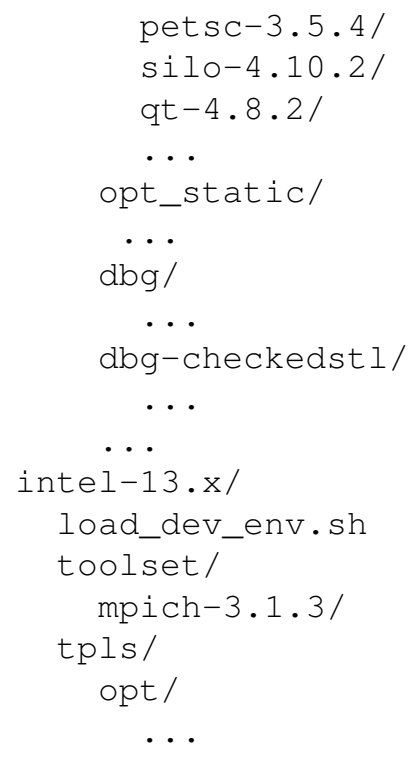

For the example in this guide, we will set:

VERA_DEV_ENV_BASE=/tools/vera

but note that any base directory can be used. This directory is where the prerequisite TPLs (see Official VERA TPL Versions) and VERA tools are deployed that are used for configuring, building, testing, and installing VERA.

For the standard GCC 4.8.3 VERA Dev Env, we set:

VERA_DEV_ENV_COMP ILER_BASE=\$VERA_DEV_ENV_BASE $/ \mathrm{gCC}-4.8 .3$

For other supported compilers (e.g. intel-13.x), other directories can be used. This directory structure keeps the compatible tools and TPLs together to maintain consistency (i.e., so we don't mix TPL builds of one compiler with TPL builds for another compiler which can otherwise cause problems in some cases).

For a given compiler set, TPLs can be installed for different configurations, such as debug (dbg), optimized (opt), or other variations (e.g. dbg-checkedstl). The standard TPL install, and the one used in this guide as an example, is:

VERA_TPL_INSTALL_DIR=\$VERA_DEV_ENV_COMPILER_BASE/tpls/opt

For the install of static TPLs, it should be:

VERA_TPL_INSTALL_DIR=\$VERA_DEV_ENV_COMPILER_BASE/tpls/opt_static

(But shared libs should be the default, see Shared versus Static Libraries.)

All of the VERA Dev Env and TPL related install tools, etc. will use the variables (shown with the typical value):

VERA Directory Env Vars:

\begin{tabular}{|l|l|}
\hline Variable & Common/Example Value \\
\hline VERA_DEV_ENV_BASE & /tools/vera \\
\hline VERA_DEV_ENV_COMPILER_BASE & \$VERA_DEV_ENV_BASE/gCC-4.8.3 \\
\hline VERA_TPL_INSTALL_DIR & \$VERA_DEV_ENV_COMP I LER_BASE/tpls/opt \\
\hline VERA_BASE_DIR & \$HOME/VERA.base \\
\hline VERA_SCRATCH_DIR & \$VERA_BASE_DIR/scratch \\
\hline VERA_DIR & \$VERA_DEV_ENV_BASE/vera-X.Y.Z-Source \\
\hline VERA_BUILD_DIR & \$HOME/VERA_BUILD \\
\hline VERA_INSTALL_DIR & /tools/Vera_installs/2015-02-06 \\
\hline
\end{tabular}


to determine a particular installation of the VERA Dev Env to use for building/testing/installing VERA components.

WARNING: When using shared libraries, one must be careful to avoid the error "RegularExpression::compile(): Expression too big." when using RPATH and when RPATH gets stripped out on install. To avoid this error, use the shortest build directory you can, like:

export VERA_BUILD_DIR=\$HOME/VERA_BUILD

\section{Installation Process}

This section gives the set of commands to run in order to install the VERA development environment and do a test build of VERA. This assumes that the entire VERA development environment will be installed starting with GCC, MPI, CMake and on up. Customizing this can be done as needed but that is not covered here. All of the sources and scripts are provided as downloads from the Internet or from given computers, or are provided as tarballs (e.g. on CDs for those who can't access outside machines from the install machines). Links to more detail are given for each step below or in the install tools themselves.

The steps are:

- Make sure the basic prerequisites are satisfied

- Determine source, scratch and install directories

- Get the base VERA and TriBITS source directories

- Install the base development environment

- Install the VERA TPLs

- Build and build/install VERA Components

- Final setup of installed VERA dev env and final cleanup

The steps in detail are given below.

\subsection{Make sure the basic prerequisites are satisfied}

Before one can install the VERA development/install environment and VERA itself, one must first:

1. Create a vera-admin Unix user, a vera-users Unix group, and the required base directories for building, testing, installing, and maintaining VERA (see Create Unix User and Group and Setup Base Directories for VERA).

2. Make sure the system can handle an installation of VERA (see System Configuration Considerations and Minimal System Package Setup).

3. If one is getting source from the VERA git repositories:

1. Set up SSH access to the ORNL machine casl-dev . ornl.gov (casl-dev for short, see SSH Setup For Accessing casl-dev and Set Up Remote SSH Tunnel).

2. Be approved to access the required protected VERA git repositories (see Requesting Access to VERA Repositories and Clone Remaining VERA Components).

\subsection{Determine source, scratch and install directories}

Set environment variables for the location of the installed/shared VERA development/install environment VERA_DEV_ENV_BASE and the base location of the VERA sources VERA_BASE_DIR to drive the install and other specified in VERA Directory Env Vars. For example: 


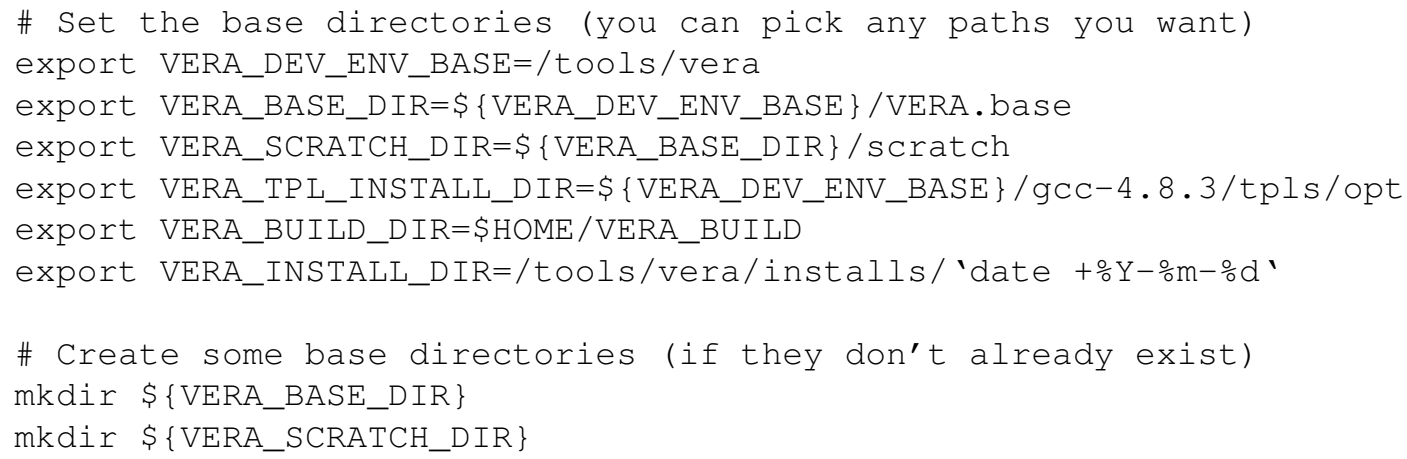

All of the other directories will be created below or automatically by the various install tools that are run.

WARNING: When using shared libraries, one must be careful to avoid the error "RegularExpression::compile(): Expression too big." when using RPATH and when RPATH gets stripped out on install. To avoid this error, use the shortest build directory you can, like:

export VERA_BUILD_DIR=\$HOME/VERA_BUILD

\subsection{Get the base VERA and TriBITS source directories}

If using a VERA tarball, do:

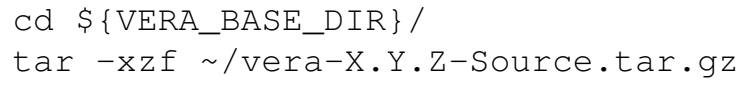

then set:

export VERA_DIR=\$ VERA_BASE_DIR $\} /$ vera-X.Y.Z-Source

If cloning the sources from casl-dev (if they are not already cloned), first setup your SSH tunnel (see Set Up Remote SSH Tunnel) if to casl-dev if needed with:

$$
\text { ssh - fN tunnelinit }
$$

then do the clones with:

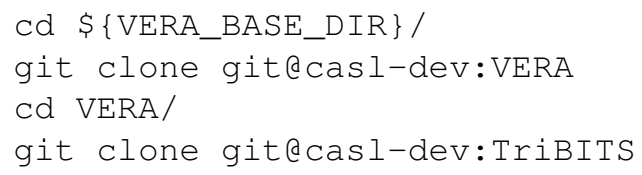

then set:

export VERA_DIR $=\$\{$ VERA_BASE_DIR $\} /$ VERA

\subsection{Install the base development environment}

To install all of the generic tools (does not include the TPLs) one can run the single command:

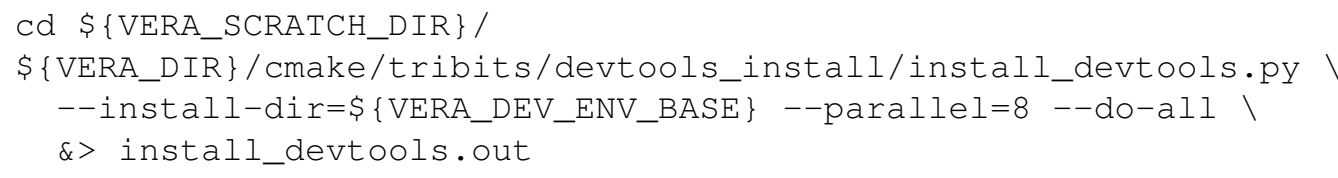

TIPS:

- Running install_devtools.py with--no-op will show what the install tool will do without actually doing anything (see --help for details). 
- Adjust --parallel=8 to the appropriate number of processes for the current machine.

- If any error occurs, look at the log file install_devtools. out to see what failed and then look at the log file it points to to see the actual errors.

- If the toolchain is being built with gec 5.X and you are encountering an error when compiling the VERA version of gec that contains:

$$
\text { ...version 'GLIBCXX_3.4.20' not found... }
$$

then the $\mathrm{c}++$ standard library must be preloaded using the following command:

$$
\text { export LD_PRELOAD=/usr/lib/libstdc++.so.6 }
$$

Note that the path to the library may be different for different systems. Also note that this environment variable will need to be added to the development and runtime environments for VERA (i.e. the \$VERA_DEV_ENV_COMPILER_BASE\}/load_dev_env.sh and the \$VERA_INSTALL_DIR\}/load_env.sh scripts).

If one does not have access to Github to be able to download the sources for the various tools, then one can obtain a compatible tarball to the git repos of the devtools sources (e.g. devtools_repos-vera-3.4.0.tar.gz), untar the tarball of the git repos in the current directory with:

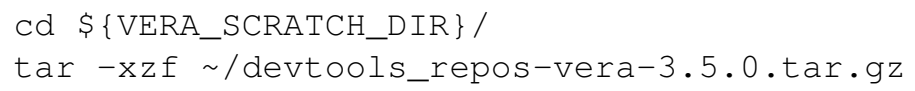

(which provides the directory \$PWD/devtools_repos/), and then additionally pass in the following argument to the above install_devtools.py script (keeping all the rest of the arguments the same):

$$
\text { --source-git-url-base=\$PWD/devtools_repos / }
$$

Once the install_devtools.py script completes, this should install GCC 4.8.3, MPICH 3.1.3, CMake 3.4.0, gitdist, and load_dev_env.sh as shown in Standard VERA Dev Env Directory Structure. For details on what can go wrong and how to deal with problems with the install, see the output from:

$$
\text { install_devtools.py --help }
$$

After a successful install, one needs to source the installed load_dev_env.sh script as:

source \$ VERA_DEV_ENV_BASE $\} / g c C-4.8 .3 / 10 a d \_d e v \_e n v . s h$

then PATH is pre-pended to find the installed programs cmake, gitdist, gcc, mpicc, ..., in the locations shown in Standard VERA Dev Env Directory Structure). Make sure you have sourced the new dev env, for example, with:

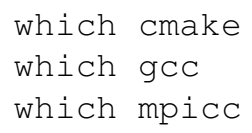

which should return:

$$
\begin{aligned}
& \ldots \text {. common_tools/cmake-3.4.0/bin/cmake } \\
& \ldots \text {.. gcc-4.8.3/toolset/gcc-4.8.3/bin/gcc } \\
& \ldots \text {.. gcc-4.8.3/toolset/mpich-3.1.3/bin/mpicc }
\end{aligned}
$$

\subsection{Install the VERA TPLs}

First, get the vera_tpls source repository and the matching version of the TPLs:

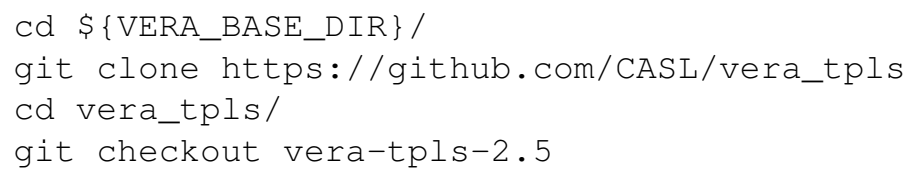


If cloning the vera_tpls repo from casl-dev, instead use:

$$
\text { git clone git@casl-dev:prerequisites/vera_tpls }
$$

Or, if one does not have access to Github or the machine casl-dev, one can obtain the tarball vera_tpls-2.5.tar.gz and untar/unzip it in the current directory using:

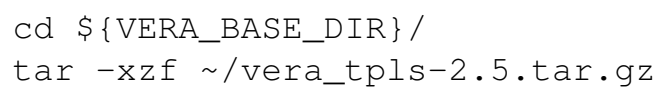

Once the directory vera_tpls for the correct version of the VERA TPLs is obtained (using one of the above methods), one can configure and build the TPLs with:

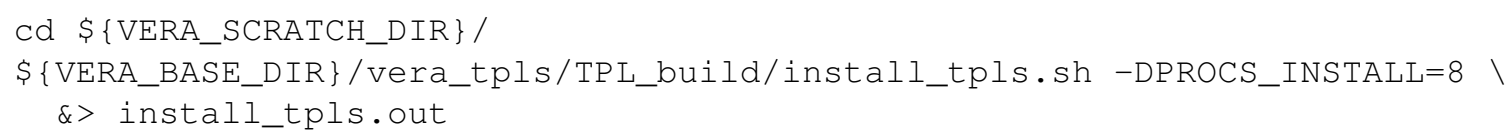

NOTE: Adjust -DPROCS_INSTALL=8 to the appropriate number of processes for the current machine.

By default, this installs a shared library version of the TPLs (see Shared versus Static Libraries). One can install a static library version passing in $-D E N A B L E \_S H A R E D=O F F$ and pass in:

-DCMAKE_INSTALL_PREFIX=\$VERA_DEV_ENV_COMPILER_BASE/tpls/opt_static

For more details, see Details on TPL Installation.

\subsection{Build and build/install VERA Components}

Finally, one must test the installed VERA Dev Env by configuring, building, testing, and installing VERA from source (installation not required).

If the VERA source was obtained from a source tarball file (see above), then all of the sources needed to build the VERA components should be in place (pointed to by $\$\left\{V E R A \_D I R\right\}$ ).

If the VERA source is obtained from the git repos on casl-dev, then the remaining VERA git repos must be cloned. First, if an SSH tunnel is necessary, first initialize the tunnel (see Set Up Remote SSH Tunnel) with:

$$
\text { ssh -fN tunnelinit }
$$

Then the remaining repos can be cloned using:

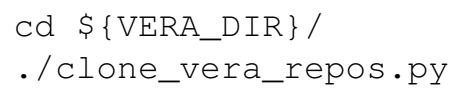

Once the source is obtained, one can configure, build, test, and install with:

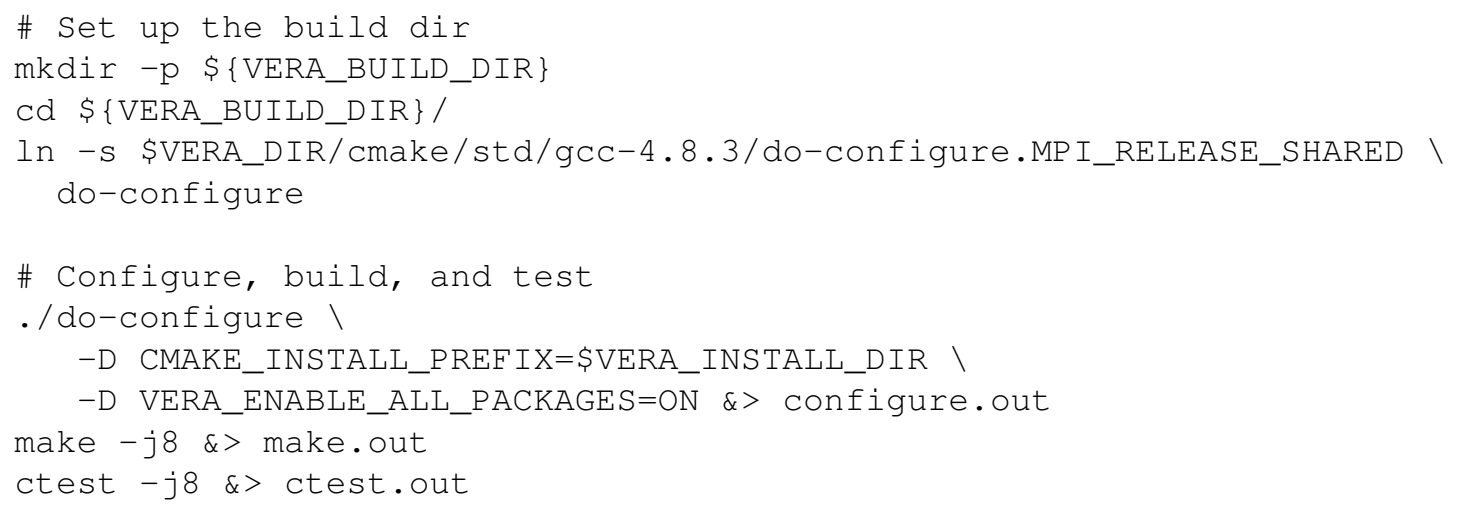

NOTE: Above shows the usage of 8 processes to build the code and run the tests. Change 8 to whatever value is appropriate for the current machine.

If all of the tests pass, i.e. the output from ctest looks something like: 


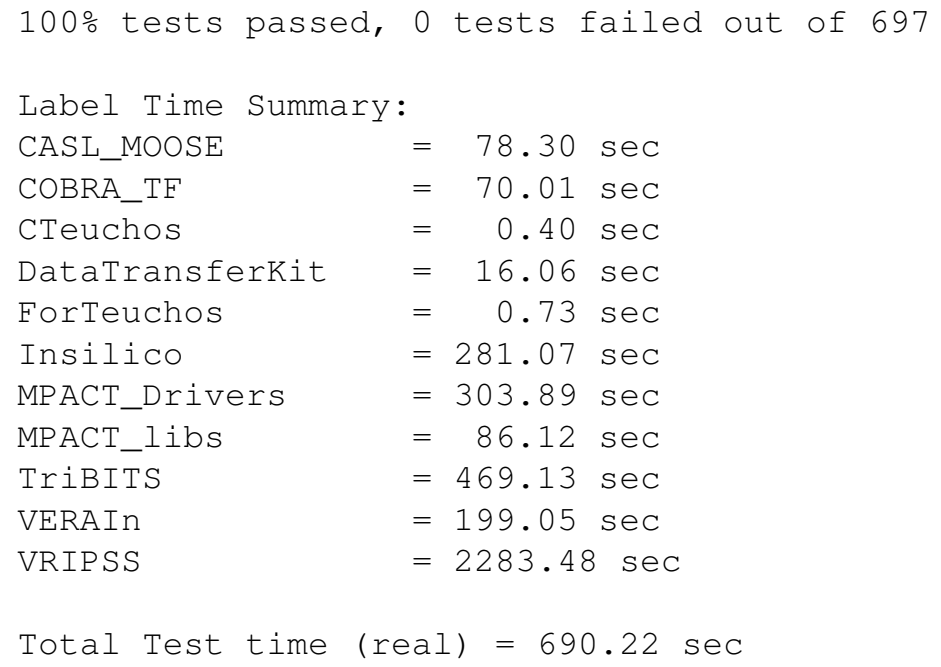

If one wants to install VERA for usage by others, this can be done with:

make -j8 install \&> make.install.out

Permissions on the installed version of VERA should be set using:

chmod chgrp -R vera-users \$VERA_INSTALL_DIR

(see Create Unix User and Group.)

After the install, see the instructions using VERA in the file:

\$VERA_INSTALL_DIR/README

In particular, one should source the script:

\$VERA_INSTALL_DIR/load_env.sh

in order to set up one's environment (i.e. PATH and other variables) so as to run the installed executables and scripts. See the online copy README.VERA (becomes the installed file README).

NOTE: The above commands build and install a shared library version of VERA (see Shared versus Static Libraries). A static library version can be installed by replacing MPI_RELEASE_SHARED with MP I_RELEASE_STATIC above. One would want to do this if installing static TPLs using -DENABLE_SHARED=OFF in Install the VERA TPLs.

\subsection{Final setup of installed VERA dev env and final cleanup}

After the VERA dev env (basic tools and TPLs) has been installed, then one must open up the directories for others to access the installed tools and libraries. Since there are no export controlled or other sensitive data contained in the installed VERA dev env, one can open it up to everyone by doing:

$$
\text { chmod }-\mathrm{R} \text { a+rX } \$\left\{V E R A \_D E V \_E N V \_B A S E\right\}
$$

When one is finished installing and testing the VERA dev env, then one can delete the scratch directory \$ $\{$ VERA_SCRATCH_DIR $\}$ if desired. However, one should save the generated $\star$. out and $\star .10 g$ files to archive details about the VERA dev env install for later reference before deleting the scratch directory.

Now that the installation process has been described, more details about the installation of the VERA dev env and VERA itself are given below. 


\section{Details on Initial Setup}

Before any of the VERA-specific prerequisites can be installed, some initial setup is required. This section describes some of the tasks that need to be performed in order to set up a machine so that it can be used to install the VERA Dev Env and then clone the VERA repositories (or just untar the VERA sources from a tarball) and build the various VERA components from source.

\subsection{Requesting Access to VERA Repositories}

If installing VERA from a release tarball, then one already has the sources. However, if installing VERA by pulling from the git repositories on casl-dev. ornl.gov, one must first be given access. Before one can access the various VERA git repositories on casl-dev. ornl. gov, one must first be given an ORNL UCAMS account, be added to the gitolite site on casl-dev, and be given explicit access to the different git repositories (in accordance to the CASL Technology Control Plan (TCP)). Contact your CASL representative to start getting this access setup. Please provide them with a list of the specific VERA git repositories (or VERA components) that one needs access.

\subsection{System Configuration Considerations}

Before work begins, an accounting of the system resources should be made. For example:

- Make sure there are enough processors available for parallel compilation. It is assumed in this document that there at least 8 cores available with which to run parallel builds, tests, etc. If that is not the case, one can reduce the parallel level as will be obvious with each command.

- Use the fastest file storage system available for holding source code, compiling, and running test cases (e.g. Try to avoid NFS-mounted directories for compilation and testing).

Meanwhile, other considerations include:

- Can the machine create SSH tunnels to download VERA and TPL repositories (if one is not building from a tarball)?

- Can the machine access the Internet (e.g. github.com)? If not, ensure that all packages needed are available in the VERA and/or TPL repositories from casl-dev using an SSH tunnel.

- Can a remote user access the machine if troubleshooting assistance is needed?

\subsection{Minimal System Package Setup}

Before proceeding to install the various VERA prerequisites, the following software packages must be installed on the system (i.e. using the systems native package manager):

- GCC gec C compiler (does not have to be a very recent version): Needed to build the official version of GCC from source. The newer GCC version will be used to builid everything else.

- GCC g++ C++ compiler (does not have to be a very recent version): Needed to build CMake from source. The newer GCC g++ version will be used to builid everything else.

- patch: Needed to support CMake.

- texinfo: The makeinfo program is needed to install GCC from source.

- GNU M4: Needed for the build of autoconf.

- Git (version 1.6.0 or newer): Needed to clone several git repositories. A newer version of git will be installed as part of the TriBITS/VERA development tools.

- python (version 2.6.6 or newer but not 3.x): Needed for the basic install scripts and some helper scripts used in VERA.

- bash: Needed for some of the shell scripts that refer explicitly to bash. 
- Perl (version v5.10.1 is what VERA is tested with but newer should work as well): Needed for building libmesh/MOOSE/Peregrine and to run the VERA input parser react $2 \mathrm{xml}$. pl.

- X11 (development libraries, not just the runtime libraries): Needed to build and install the required VERA TPL QT.

- ZLIB (development libraries, not just the runtime libraries): Needed to build and install the required VERA TPL HDF5.

For the exact packages that one needs to install on different systems, see Minimal System Package Setup on Various Systems.

There are many other software packages that one needs but the rest should already be installed on any reasonable Linux/Unix system.

\subsection{SSH Setup For Accessing casl-dev}

In order to access the VERA repositories on casl-dev. ornl.gov, one must set up public/private SSH keys and then the public SSH must be registered with the gitolite system that is used to manage the VERA git repositories. In this guide, we use <ucams-id> to signify the user's 3-char ORNL UCAMS ID.

If the machine casl-dev (casl-dev.ornl.gov) is not directly reachable from your machine (referred to as <your-machine> in this document), you will first need to set up a remote SSH tunnel to casl-dev as described in Set Up Remote SSH Tunnel.

First, on the given machine, one sets up public/private SSH keys (if not already existing) as:

$$
\$ \mathrm{~cd} \sim / \mathrm{ssh} \& \& / \text { usr/bin/ssh-keygen -t rsa -b } 1024
$$

Several prompts will appear. The defaults should be accepted with three strikes of the $<$ ENTER $>$ key.

The public key just created, /.s.sh/id_rsa.pub, must then be sent by email to casl-vri-infrastructure@casl.gov in order to be registered with one's account in the gitolite system on casl-dev. In addition, you must be approved the VERA git repositories before one's key can be added.

If one is not on the ORNL network with direct access to the machine casl-dev, then one will need to open the SSH tunnel to casl-dev using:

$$
\text { ssh - fN tunnelinit }
$$

(See Set Up Remote SSH Tunnel.)

After one's public SSH key has been registered with gitolite on casl-dev (and the SSH tunnel to cas I-dev has been established if needed), then one can test to see if one has repo access by running the command:

$$
\text { ssh gitecasl-dev info }
$$

This command should return the list of git repositories for which one has access. At the very minimum, this should return:

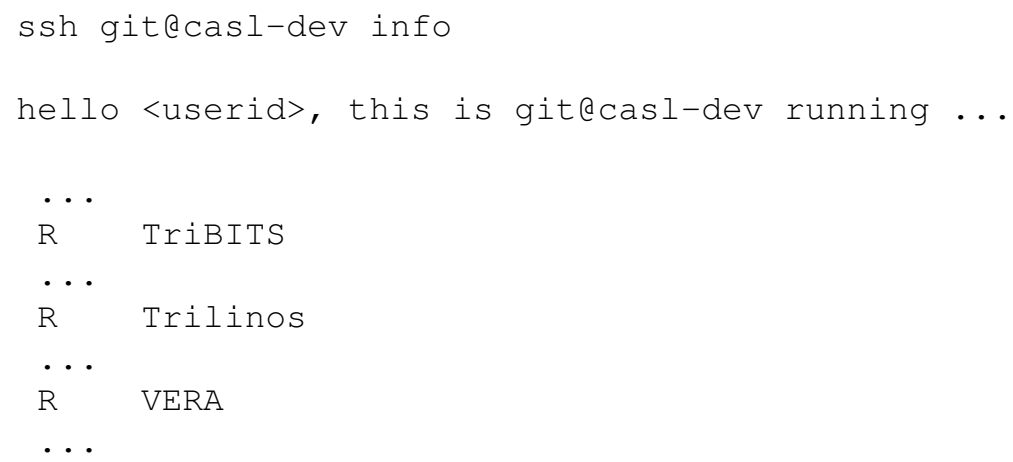

If this command does not work without a password challenge, then something is wrong and no repositories will be able to be cloned.

Access to other repositories requires being explicitly added to the appropriate gitolite groups (again, contact your CASL representative). 


\subsection{Create Unix User and Group}

In order to properly administer and protect VERA installations, it is recommended to set up the following:

- A vera-admin user: Unix user account specifically for maintaining VERA installations.

- A vera-users group: List of Unix users that have access permission and need-to-know for running the installed VERA components.

NOTE: The list of users added to vera-users must have been given explicit permission to access all of the installed VERA components. If one is not sure who can be in this list, please contact support@casl.gov or some other responsible CASL representative for guidance.

\subsection{Setup Base Directories for VERA}

Once the vera-admin user account has been created, the \$ $\{$ VERA_DEV_ENV_BASE $\}$ and \$ $\{$ VERA_INSTALL_DIR \} directories need to be created by the root user or a user with sudo privileges. Then ownership of these directories needs to be transfered over to the vera-admin user. This can be performed using the following commands:

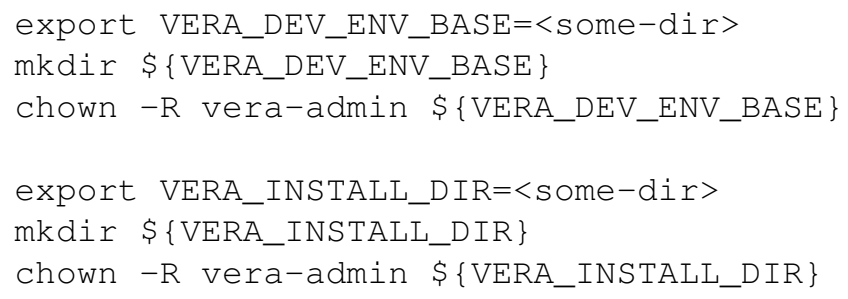

To reduce the need for root or sudo access during the installation process, a $\$$ VERA_ROOT_DIR $\}$ can be used which will contain the following directories:

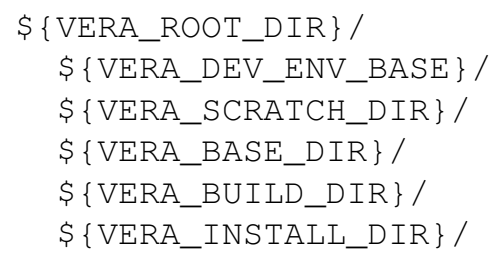

By using this directory structure, someone with root or sudo access only needs to perform the following operations:

- Make sure all of the required system packages have been installed.

- Create the vera-admin user.

- Create the vera-users group.

- Create the \$ $\{$ VERA_ROOT_DIR $\}$.

- Transfer ownership of \$\{VERA_ROOT_DIR $\}$ over to vera-admin.

Once these actions have been performed, the vera-admin user will have all of the necessary permissions to build, test, install, and maintain the VERA installation.

\section{Details on TPL Installation}

A standard installation of the VERA TPLs can be performed using the command:

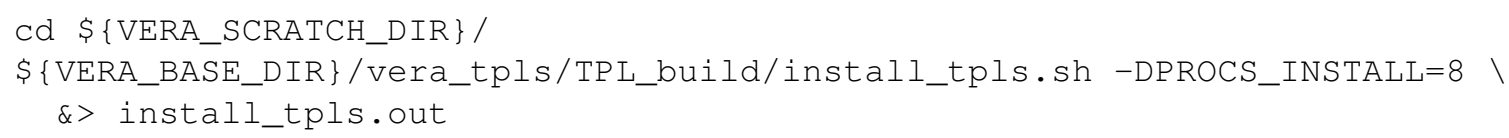


as described in Install the VERA TPLs. However, this is just a thin little shell script that uses a CMake ExternalProject build of the TPLs.

The TPL build system can be pulled by using:

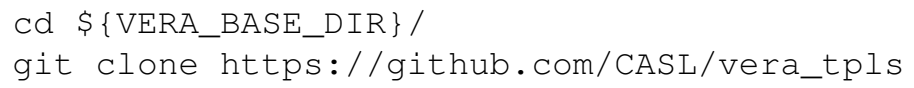

when pulling from github. When pulling from cas l-dev using $\mathrm{SSH}$, use:

Cd \$ $\{$ VERA_BASE_DIR $\} /$

git clone gitecasl-dev:prerequisites/vera_tpls

The shell script install_tpls.sh just creates a CMake binary directory:

\$ $\{$ VERA_SCRATCH_DIR $\} / T P L \_b u i l d /$

and then runs CMake pointing to the CMake ExteranlProject directory:

\$ VERA_BASE_DIR \}/vera_tpls/TPL_build/

This TPL_build CMake project accepts a number of CMake cache variables which include:

- CMAKE_BUILD_TYPE: Release or Debug

- ENABLE_SHARED: ON means that only shared * . so libs will be installed while OFF means that only static * . a libs will be installed.

- CMAKE_INSTALL_PREFIX: Base path to TPL install location. The TPLs are installed under this directory, one subdirectory for each TPL.

- TPL_LIST: List of TPLs to install. This can be a comma separated list. The full list of TPLs (and the default value for this variable) is:

ZLIB; HDF 5; LAPACK; QT; PETSC; SLEPC; SILO; BOOST

Any subset of TPLs can be listed and will be installed instead.

- PROCS_INSTALL: Number of processors to use to build each TPL. At least 4 is recommended due to the lengthy BOOST and QT build times. Last Line Path to "vera_tpls/TPL_build repository sub-directory

The install tool:

\$ $\{$ VERA_BASE_DIR\}/vera_tpls/TPL_build/install_tpls.sh

is a bash script that sets all of the defaults needed to do the basic build, including compiler options, etc. But as shown in Install the VERA TPLs, one can pass in any CMake cache variable and it will override the defaults set in install_tpls.sh. For example, to install static libraries in addition to shared libraries and use 16 processes, one can use:

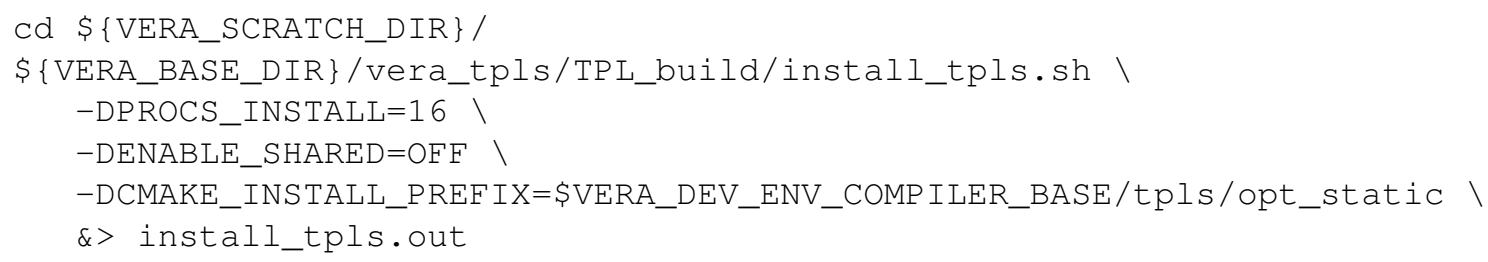

After the above install script finishes calling cmake to configure the TPL build, it just calls:

make

to drive the build and install process.

One can just examine the script install_tpls.sh to see what it does and can therefore make needed modifications to the install process and run the commands manually if needed. 


\section{Details VERA Component Build, Test, and Installation}

Once the VERA prerequisites (i.e., compilers, TPLs, other tools) have been installed, one needs to clone the remaining VERA git repositories for the desired components (if working from the version-controlled source), set up a build configuration, build, test, and finally install. If working from an untarred (release) tarball, no extra git clones are needed. All of the required source will already be in place.

\subsection{Load VERA Dev Env}

Before one can configure, build, test, and install any VERA component software, the installed VERA Dev Env must first be loaded into the users shell. If not already loaded, then run:

source \$\{VERA_DEV_ENV_COMPILER_BASE\}/load_dev_env.sh

\subsection{Clone Remaining VERA Components}

When working from a tarball distribution of VERA, there is nothing left to clone so one can skip this section.

However, if working from the version-controlled sources, the remaining VERA git repositories can be cloned, for example, as:

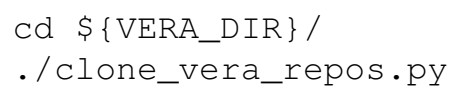

NOTE: The exact list of repositories that one needs to clone greatly depends on what VERA components with what functionality one desires or needs from VERA. Such information is not provided in this document. Ask your CASL VERA contact about what repositories you need to clone for your needs.

NOTE: One must be part of the gitolite group for gitecasl-dev that protects a repository or when one clones one will get an error message like:

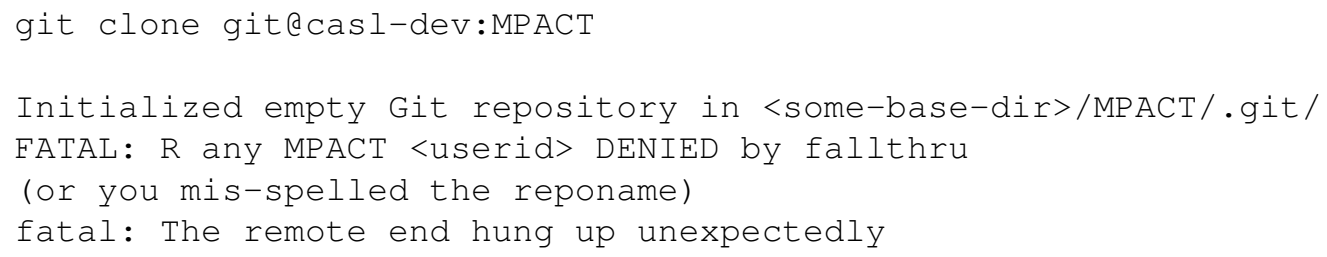

where $<$ some-base-dir $>$ and $<$ userid $>$ are replaced with the local base directory and the gitolite user account name under git@casl-dev. To see if one has misspelled the repo or if one just does not have permission, run:

$$
\text { ssh gitecasl-dev info }
$$

If the git repository that one is trying to clone is not listed in the output from this command, then one don't have permissions to clone the given git repository.

\subsection{Checking Out a Specific Version of VERA}

When working from a tarball distribution of VERA, the version is fixed in which case, one can skip this section. However, when working from the git repositories, various versions of VERA can be accessed.

The most recent version of VERA can be pulled using:

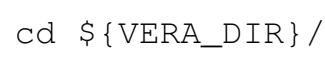


This will pull the most recent version of VERA (at that moment) from the official development cas l-dev/master branches. While a rigorous almost continuous integration process ensures that all of the basic automated tests pass before anything is pushed into the casl-dev/master branches, mistakes do occur and there may be some more detailed acceptance tests that may not run successfully on any particular version of VERA at any moment for what is in casl-dev/master.

Therefore, to reduce the probability of the customer pulling a defective version of VERA components for their usage, it is recommended that more specific versions of VERA be pulled that have undergone more testing (both more expensive automated acceptance tests run nightly and weekly as well as some larger manually run tests in some cases). Checking out a specific version of the VERA repositories is accomplished using the gitdist tool and a VERARepoVersion.txt file. A VERARepoVersion.txt file is created whenever VERA is configured from local git repositories and that file is written to the VERA build tree and it gets installed in the base install tree. Every automated VERA build/test posted to the VERA CDash server includes exact git version information in the generated VERARepoVersion.txt file. For a subset of VERA repos, a VERARepoVersion. txt file looks like:

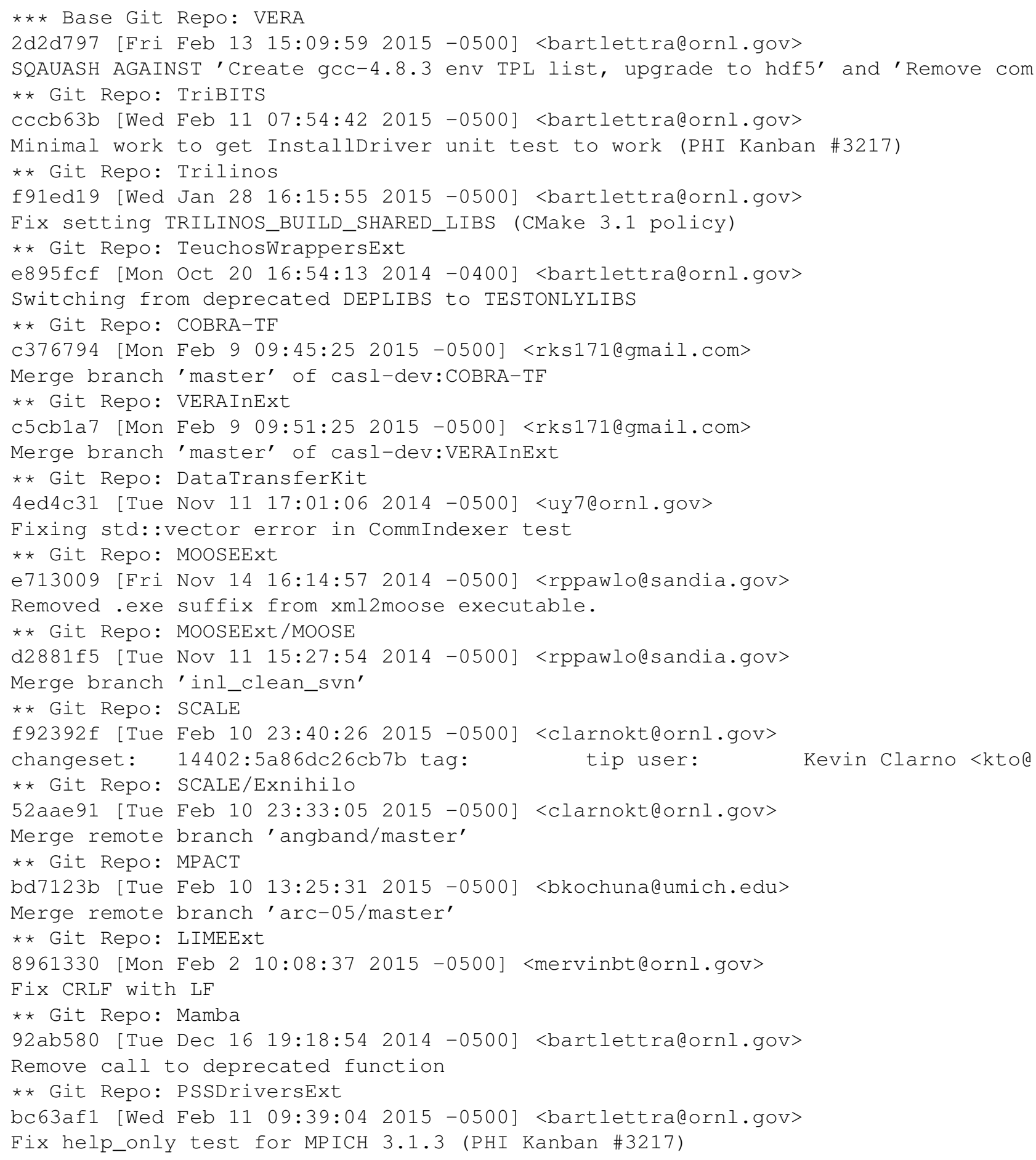

Before updating to a specific version of VERA, a CASL representative will provide the customer a specific 
VERARepoVersion.txt file, typically named VERARepoVersion. <newdate $>$. txt (for some specific date $<$ newdate $>$ =YYYY-MM-DD), which the customer can use to checkout that specific version with:

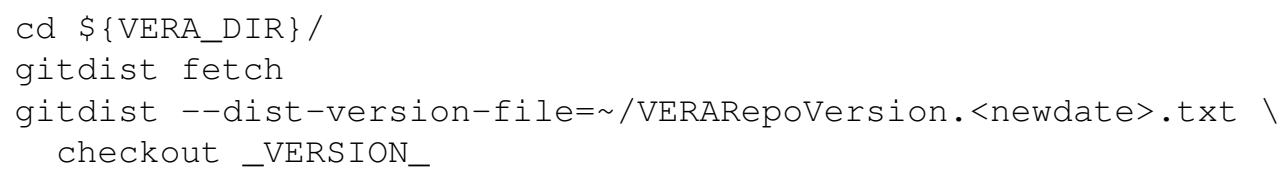

(see gitdist - -help for more details.)

This will create a "detached head" state for the local VERA git repos where each repo will be at the exact commit listed in the VERARepofileVersion. <newdate>.txt file. Here is the message that you might get from each of the repos:

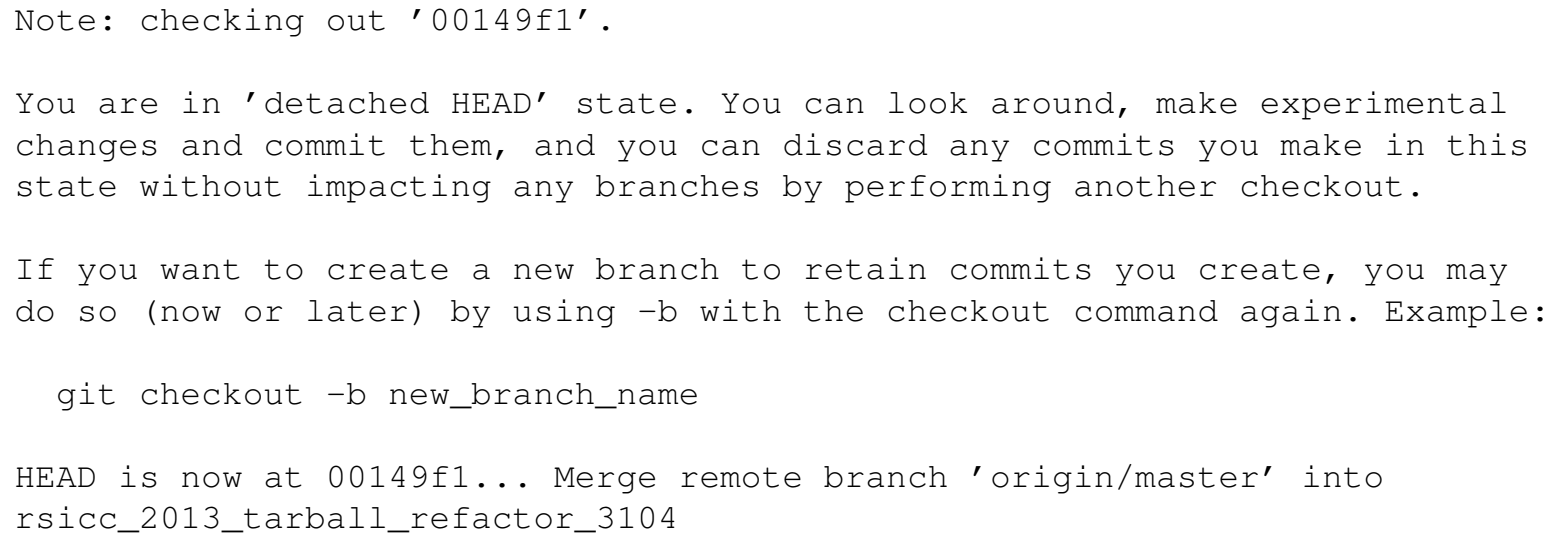

This is not a troublesome state for the purposes of just building the source.

Also, using the VERARepoVersion. txt file for a previous install, one can see what repos have been changed and what commits have been added. For example, to compare to an older install in:

$$
\text { \$ VERA_DEV_ENV_BASE }\} / \text { vera/<olddate }>/
$$

one could compare to the new recommended version by running:

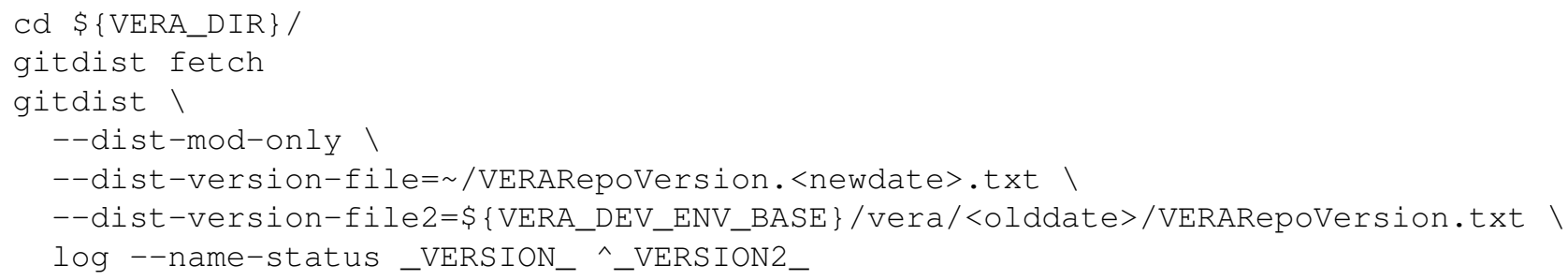

Many other types of git commands are possible where one or two of the repo versions can be supplied through a VERARepoVersion.txt file.

\section{Details on Finalizing VERA Dev Env Installation}

Once one has finished installing the VERA prerequisites consisting of the compilers, OpenMPI, and other tools and a complete set of TPLs shown in Standard VERA Dev Env Directory Structure and one is finished testing the installs by building and testing needed VERA components, one just needs to fix up permissions on the installed files and directories.

The directory permissions for the Unix tools, compilers, and TPLs can be opened up for all to use. This can be accomplished with the following command:

$$
\text { chmod }-\mathrm{R} \text { a+rX <some-dir }>
$$

For example, world readable permissions can be assigned using the following commands: 


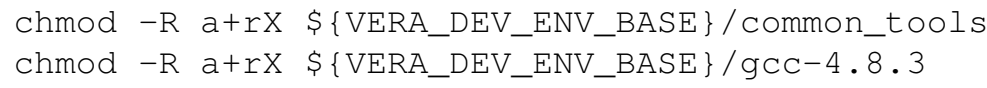

That should allow anyone to read any of the installed files and directories (but not modify them and mess them up).

If the owning user is not vera-admin, change the owning user to avoid accidental modifications by the original installer using the following command:

chown -R vera-admin <some-dir>

for example, if the original installer is not the current owning using, then the following commands can be run to transfer ownership over to the vera-admin user:

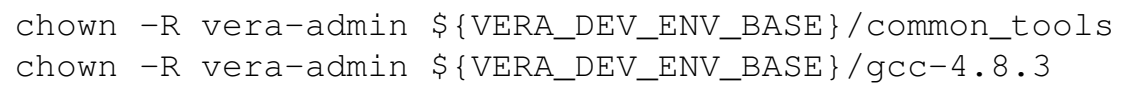

This will avoid problems with accidental modifications to the installed directories by the original installer.

As an optional final step, to clean up disk space, one can delete the scratch space and TPLs source repo by doing:

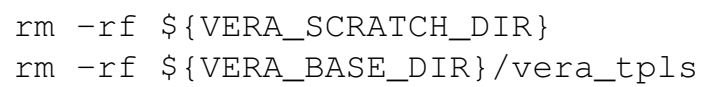

WARNING: One should only remove these directories after one is sure that the VERA dev env is correctly installed and that the necessary dependent VERA components are building and running correctly (at least related to the installed VERA dev env).

All that should be left locally would be the local VERA source and build tree:

$$
\text { \$VERA_BASE_DIR }\} /
$$

which can be used to clone and build VERA components using the installed VERA Dev Env. However, if VERA will no longer be built under this directory, then it can be removed as well with:

rm -rf \$VVERA_BASE_DIR\}

All that would left would be the install of the VERA dev env under $\{\{$ VERA_DEV_ENV_BASE $\} /$.

After all of this, the VERA Dev Env would be considered successfully installed and now users of the dev env just need to source the script, for example:

source \$ VERA_DEV_ENV_BASE\}//gCc-4.8.3/load_dev_env.sh

(in their .bash_profile file for instance).

\section{Details on Installing VERA}

Once the VERA Dev Env is installed on a system and loaded in the user's shell environment, then anyone with access to the VERA sources (either through a tarball or through the cloned git repositories) can configure, build, test, and install the VERA components.

Information on the installation directory layout is found at:

\$ $\{$ VERA_DIR\}/doc/install/README.VERA

\subsection{Get Source For VERA Components To Install}

Getting the sources for the VERA components to install is identical to getting them to install and test the VERA Dev Env. This can be done simply with:

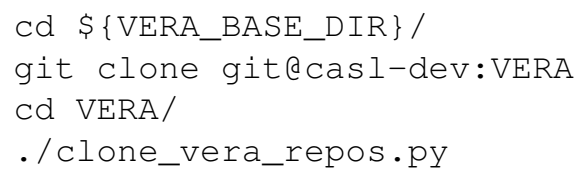




\subsection{Configure, Build, And Test VERA Components To Install}

To set up to build, test, and install various VERA components by end users, one must first select a configuration setting. For most systems, shared libraries should be preferred since they typically use up less disk space.

When installing VERA, it is important to build in a very shallow build directory. For example, using local scratch space to set up a build directory:

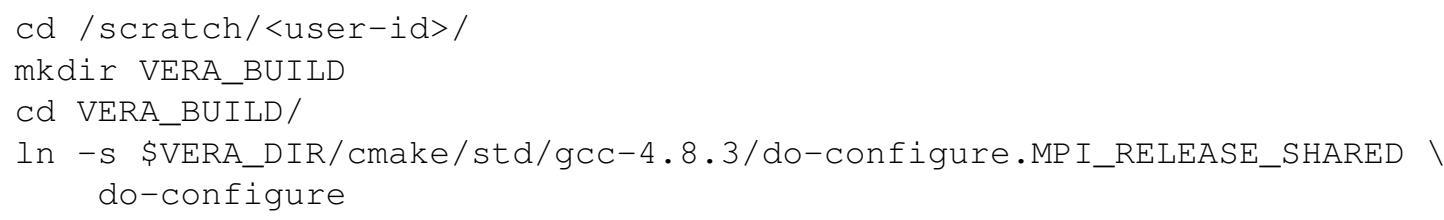

If you don't use a shallow build directory, then CMake may fail to replace the RPATHs in the executables due to strings being too long.

Once a do-configure script is set up, one just needs to configure pointing to the final install location. Assuming one will install into:

export VERA_INSTALL_DIR=/tools/vera_installs/'date + $\frac{\circ}{}$ - $\frac{\circ}{\circ}-\frac{\circ}{\circ}$ '

one would configure with:

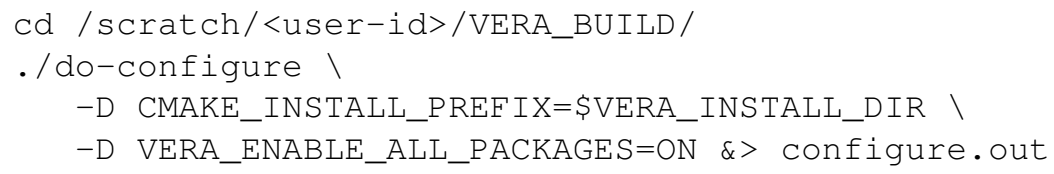

Assuming the configure passed, one would build and test using:

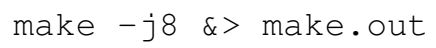

(see Build and build/install VERA Components).

All of the tests should pass. If they do not, please send email to support@cas l.gov giving the tail of ctest.out and a copy of your do-configure script.

\subsection{Install Built VERA Components}

Before running make install, in order to protect VERA appropriately, one may need to set up the base directory for the install as:

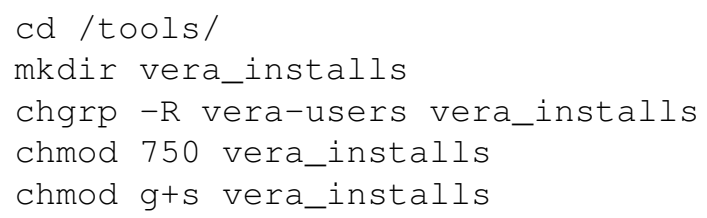

(see the vera-users group described in Create Unix User and Group.)

After a successful configure, build, and test has been performed, an install is simply performed as:

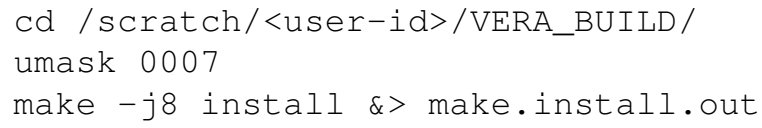

Setting umask 0007 will ensure that only the vera-users group (or whatever it is called on the given system) will have access to the installed VERA software.

This should set up an installation directory that looks like: 


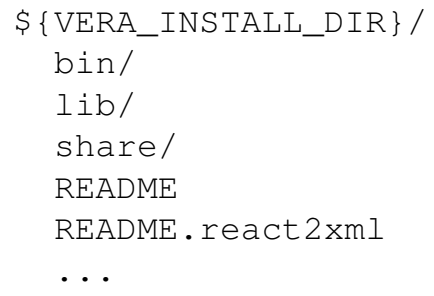

\subsection{Documentation For Installed VERA Components}

Once installed, information on how to access the installed VERA components along with their documentation and examples is found in the file:

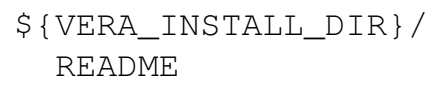

\section{Appendix}

\subsection{Set Up Remote SSH Tunnel}

In order to access the Git repositories on casl-dev. ornl.gov when outside of the ORNL network, a SSH tunnel must be set up through login1. ornl. gov. This requires the user to have an active UCAMS account with the 3-char <ucams-id>. Once established, this SSH tunnel will set up a machine name called casl-dev on the local machine that can then be used in Git commands.

In one's home directory, create the file:

$$
\sim / \text { ssh/config }
$$

which contains:

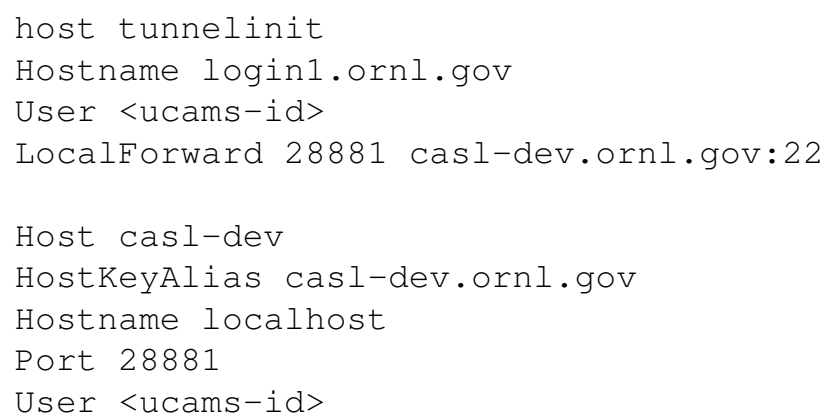

Please make sure to change the above port numbers to not conflict with other ports being used on the system or other SSH tunnels. If multiple users use the same port numbers there will be collisions, or the host machine will disallow the connection altogether.

To set up the SSH tunnel, in any terminal, type the command:

$$
\text { ssh -fN tunnelinit }
$$

You will be prompted for a PASSCODE, this is your pin+6digits from your SecurID token. This will return to the command-line prompt and then one can test open a SSH connection to cas l-dev as:

$$
\text { ping casl-dev }
$$

The SSH tunnel will stay open for some amount of time, longer if it is being actively used. However, it may be important in some cases to ensure that the tunnel is closed before logging off or doing other tasks. If a user creates a SSH tunnel, the user should be able to close the SSH tunnel. Since the SSH tunnel is in the background the user should use the following command to find the ssh tunnel process.: 
ps aux | egrep ssh -fN tunnelinit

This will return something along the lines of:

$$
\text { <ucams-id> } 105350.3 \quad 0.0 \quad 66032 \quad 3804 \text { ? Ss } 11: 08 \quad 0: 19 \text { ssh -fN tunnelinit }
$$

The user can then use the kill command to end the tunnel process:

kill $-9<$ process number $>$

in this case 10535, the process number will always be the second item in the returned fields from the ps aux command.

This will close the SSH tunnel.

\subsection{Minimal System Package Setup on Various Systems}

Package managers on different systems have different names for the packages they install that are needed described in Minimal System Package Setup.

The following table gives the names of the packages that need to be installed on three different machines where VERA has been installed in the past. In addition, the last column specifies the executable that should be in the system's path provided that the package is installed. Testing for the existence of these executables can be accomplished with the which command. For example:

which gCC

may return:

/usr/bin/gcc

Note that if a path to the executable is not returned by the which command, then the package is not installed.

\begin{tabular}{|c|c|c|c|c|}
\hline Package & CentOS 6.6/Redhat 6.4 & Ubuntu 14.04.1 & openSUSE 11.4 & Executables \\
\hline GCC C Compiler & gcc & gcc & gcc & $\mathrm{gcc}$ \\
\hline GCC C++ Compiler & gcc-c++ & $\mathrm{g}++$ & $\mathrm{g}++$ & gcc-c++ \\
\hline GNU M4 & $\mathrm{m} 4$ & M4 & M4 & $\mathrm{m} 4$ \\
\hline GNU texinfo & texinfo & texinfo & & makeinfo \\
\hline Git & git & git & git & git \\
\hline Python & python & Python-minimal & python & python \\
\hline Bash & bash & bash & bash & bash \\
\hline Perl & perl & perl-base & perl & perl \\
\hline X11 (dev) & libX11-devel & libx11-dev & & \\
\hline Xorg (dev) & xorg-x11-server-devel & xorg-dev & & \\
\hline Zlib (dev) & zlib-devel & zlib-dev & & \\
\hline
\end{tabular}

Note that there are no executables for the X11, Xorg, or Zlib development libraries. To verify that these packages are installed, one can try and look for a required library (in the user's LD_LIBRARY_PATH) or a required include file or directory in / usr/include. Suggested files/folders to check for to verify installation of the X11, Xorg, and Zlib development libraries are provided in the following table.

\begin{tabular}{|l|l|l|}
\hline Package & usr/lib or /usr/lib64 & /usr/include \\
\hline X11 (dev) & libX11.so & X11 \\
\hline
\end{tabular}

... continued on next page 


\begin{tabular}{|l|l|l|}
\hline Package & usr/lib or /usr/lib64 & /usr/include \\
\hline Xorg (dev) & & xorg \\
\hline Zlib (dev) & libz.so & zlib.h \\
\hline
\end{tabular}

On CentOS and Redhat systems, the command yum install <package> installs a package, for example:

sudo yum install gcc-c++

On Ubuntu systems, the command apt-get install <package> installs a package, for example:

sudo apt-get install g++

On SUSE and openSUSE systems, the command zypper install <package> installs a package. For example:

sudo zypper install gcc-C++

Note that someone with sudo privileges must install these packages since they get installed into the base system directories. However, it is possible to use some package managers to install missing packages to a different location that does not require root or sudo access but that is beyond the scope of this document (consult the documentation for the package manager on your system).

\subsection{Official VERA TPL Versions}

The source code for the official TPL versions that are installed using the install_tpls.sh tool are storied in the vera_tpls git repository and the current official versions are shown in the below table.

\begin{tabular}{|l|l|}
\hline Third Partly Library (TPL) & Version \\
\hline LAPACK & 3.3 .1 \\
\hline Boost & 1.55 .0 \\
\hline ZLib & 1.2 .7 \\
\hline HDF5 & 1.8 .10 \\
\hline PETSc & 3.5 .4 \\
\hline SLEPC & 3.5 .4 \\
\hline Silo & 4.10 .2 \\
\hline QT & 4.8 .2 \\
\hline
\end{tabular}

The exact version of TPLs matching this particular version of VERA is always given by the git tag for the vera_tpls repo given in the section Install the VERA TPLs.

\subsection{Shared versus Static Libraries}

The default install of the VERA TPLs in Install the VERA TPLs and VERA itself in Build and build/install VERA Components use shared libraries. However, these instructions also describe how to install static libraries. In general, one should prefer shared libraries over static libraries on most platforms as shared libraries use less disk space, link faster, and allow for quick bug fixes and simpler upgrades. But if RPATH is not written into the executables and shared libraries, then one will have to set LD_LIBRARY_PATH pointing to the location of the correct shared libraries.

However, some systems (especially some HPC machines) either require or strongly recommend the usage of static libraries. In addition, static libraries are more self-contained and are less sensitive to the machine environment from which they run and they never have RPATH issues. But executables built using static libraries take up far more disk space and take longer to link. 